・研究简报・

\title{
低聚噻吩衍生物单体的合成及其聚合物薄膜的电致变色性能
}

\author{
姜月 ${ }^{a}$ 王 娟 ${ }^{a}$ 关 丽 ${ }^{a, b}$ 钟一平 ${ }^{a}$ 刘 平 ${ }^{*}, a$ 邓文基 ${ }^{c}$ \\ ( ${ }^{a}$ 发光材料与器件国家重点实验室 华南理工大学材料科学研究所 广州 510640)

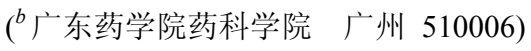 \\ (c华南理工大学应用物理系 广州 510640)
}

\begin{abstract}
摘要 合成了两种低聚噻吩衍生物单体: 2,3':4',2"-三噻吩(I3T)和 5,5"-二醛基-2,3':4',2"-三噻吩(OHC-I3T-CHO). 通过电 化学方法对单体 I3T 和 OHC-I3T-CHO 进行了聚合, 制备了相应的聚噻吩衍生物. 研究了聚噻吩衍生物薄膜的电致变色 性能. 当外加一定电压时, 基于单体 $\mathrm{I} 3 \mathrm{~T}$ 的聚噻吩衍生物薄膜可以在淡黄色和淡蓝色之间发生可逆的颜色变化, 基于单 体 OHC-I3T-CHO 的聚噻吩衍生物薄膜能在红色和墨绿色间发生可逆的颜色变化.
\end{abstract}

关键词 低聚噻吩衍生物单体; 电化学聚合; 聚噻吩衍生物薄膜; 电致变色性能

\section{Preparation and Electrochromic Properties of Polythiophene Deriva- tive Films Based on Oligothiophene Derivative Monomers}

\author{
Jiang, Yue ${ }^{a} \quad$ Wang, Juan ${ }^{a} \quad$ Guan, Li ${ }^{a, b} \quad$ Zhong, Yiping ${ }^{a} \quad$ Liu, Ping*,a $\quad$ Deng, Wenji \\ ( ${ }^{a}$ State Key Laboratory of Luminescent Materials and Devices, Research Institute of Materials Science, \\ South China University of Technology, Guangzhou 510640) \\ $\left({ }^{b}\right.$ College of Pharmacy, Guangdong Pharmaceutical University, Guangzhou 510006) \\ ( ${ }^{c}$ Department of Applied Physics, South China University of Technology, Guangzhou 510640)
}

\begin{abstract}
Oligothiophene derivative monomers, 2,3':4',2"-terthiophene (I3T) and 5,5"-biformyl-2,3':4',2"terthiophene (OHC-I3T-CHO), were synthesized. The corresponding polythiophene derivatives based on I3T and OHC-I3T-CHO monomers were prepared by electropolymerization. The electrochromic properties of polythiophene derivative films were investigated. When the voltage were applied between the ITO working electrode and the platinum wire counter electrode, the I3T film showed reversible, clear color changes from light yellow to light blue, while the OHC-I3T-CHO film showed reversible, clear color changes from red to greenish black.
\end{abstract}

Keywords oligothiophene derivative monomer; electropolymerization; polythiophene derivative film; electrochromic property

聚噻吩及其衍生物是一类重要的有机共轭高分子 功能材料. 从理论上说, 聚噻吩被当作研究非简并态导
电高分子电子迁移的模型; 从应用上来说，由于噻吩芳 杂环对氧气和湿度都有较高的稳定性, 使其衍生物比其

\footnotetext{
* E-mail: mcpliu@scut.edu.cn

Received September 9, 2011; accepted November 30, 2011.

Project supported by the National Natural Science Foundation of China (Nos. 20674022, 20774031 and 21074039), Doctoral Fund of Ministry of Education of China (No. 20090172110011) and Science and Technology Project of Guangdong Province of China (Nos. 2006A10702003, 2009B090300025 and 2010A090100001).

国家自然科学基金(Nos. 20674022，20774031，21074039)、教育部博士点基金(No. 20090172110011)、广东省科技计划项目(Nos. 2006A10702003, 2009B090300025, 2010A090100001) 资助项目.
} 
它芳杂环衍生物更易被合成, 同时, 聚噻吩的掺杂态和 去掺杂态都具有良好的环境稳定性. 因此, 聚噻吩及其 衍生物在有机半导体领域具有广泛的应用前景. 近些年 来, 利用电化学方法制备聚噻吩及其衍生物得到了研究 者的高度关注 ${ }^{[1 \sim 5]}$.

电致变色现象是指材料或体系在外加电场作用下 发生可逆电化学反应而使物质的光学性能(透射率、反 射率或吸收率)在各种波长范围内产生稳定、连续可逆 变化的现象, 在外观上表现为颜色和透明度的可逆变 化. 电致变色材料被认为是目前最有应用前景的智能材 料之一, 用电致变色材料组装的电致变色器件可以应用 于智能窗、显示器以及国防军事等领域. 目前, 电致变 色材料主要是以氧化铱、氧化铑、氧化铇等无机过渡金 属氧化物为主, 用无机电致变色材料制成的无机电致变 色器件已经部分转化为商品, 得到实用. 而有机电致变 色材料及其器件还处于研究阶段 ${ }^{[6]}$, 远未达到实用. 但 无机电致变色材料及器件的制造成本较高, 而且某些无 机电致变色材料还具有不可降解的毒性. 另外, 无机电 致变色材料不宜柔性加工. 与无机电致变色材料相比, 有机电致变色材料具有化合物结构可设计, 纯度高, 材 料质量轻, 加工性能优, 毒性小, 制造成本较低等优点. 因此, 研究有机电致变色材料及其器件是一个非常重要 的课题.

本论文中, 我们以发展新型有机电致变色材料为目 的, 合成了两种低聚噻吩衍生物单体: $2,3^{\prime}: 4^{\prime}, 2^{\prime \prime}$-三噻吩 (I3T) 和 5,5"-二醛基-2,3':4',2"-三噻吩 (OHC-I3T-CHO). 通过电化学方法对单体 I3T 和 OHC-I3T-CHO 进行了聚 合, 制备出相应的聚噻吩衍生物, 研究了聚噻吩衍生物 薄膜的电致变色性能.

\section{1 实验部分}

\section{1 原料与试剂}

所用溶剂采用通常的方法纯化和干燥; 2-硼酸噻 吩、3,4-二溴噻吩、三氯氧磷 $\left(\mathrm{POCl}_{3}\right)$ 、二(三苯基膦)二 氯化钯 $\left[\mathrm{Pd}\left(\mathrm{PPh}_{3}\right)_{2} \mathrm{Cl}_{2}\right]$ 、高氯酸四丁基胺(TBAP)均为 Aldrich 公司产品, 直接使用.

\section{2 测试仪器}

质谱仪(Esquire HCT PLUS, 德国 Bruker 公司); 核 磁共振仪(AVAECE400, 德国 Bruker 公司); 红外光谱仪 (VECTOR, 德国 Bruker 公司); 单晶衍射仪(D/max-IIIA, 日本理学公司); 电化学工作站(CHI750A 型, 上海辰华 仪器公司).

\section{3 低聚噻吩衍生物单体的合成}

单体 I3T 和 OHC-I3T-CHO 的合成路线如 Scheme 1
所示.
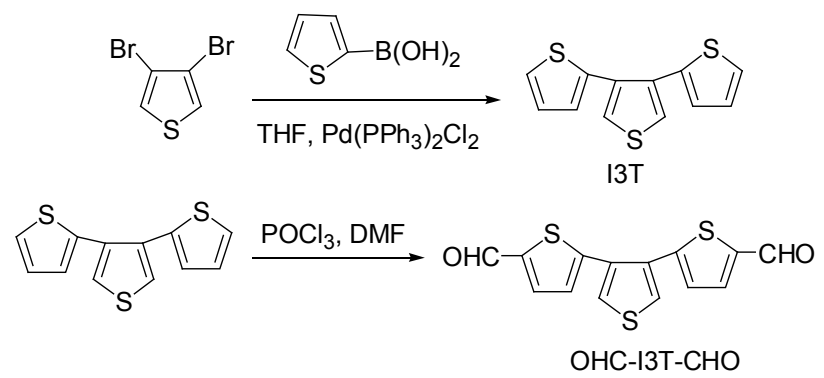

图式 1 低聚噻吩衍生物单体 I3T 和 OHC-I3T-CHO 的合成

Scheme 1 Synthesis of oligothiophene derivative monomers, I3T and OHC-I3T-CHO

\subsubsection{2,3':4',2"-三噻吩(I3T)的合成}

在 $\mathrm{N}_{2}$ 保护下, 先将四氢呋喃(THF)加入到三颈瓶中, 然后将 2-硼酸噻吩、3,4-二溴噻吩、 $\mathrm{K}_{2} \mathrm{CO}_{3}$ 水溶液依次 加入此三颈瓶中, 最后加入催化剂 $\mathrm{Pd}\left(\mathrm{PPh}_{3}\right)_{2} \mathrm{Cl}_{2}$; 充分 搅拌条件下加热回流 $40 \mathrm{~h}$, 反应结束后加入饱和 $\mathrm{NH}_{4} \mathrm{Cl}$ 溶液, 然后用二氯甲烷萃取; 再用饱和 $\mathrm{NaCl}$ 洗涤, 最后 再用二氯甲烷萃取, 萃取液经无水 $\mathrm{Na}_{2} \mathrm{SO}_{4}$ 干燥后减压 蒸馏除去二氯甲烷得到粗产物. 用石油醚作洗脱液, 粗 产物经硅胶柱分离提纯得到白色晶体，产率为 $76 \%$.

1.3.2 5,5"-二醛基-2,3':4',2"-三噻吩(OHC-I3T-CHO) 的 合成

在 $\mathrm{N}_{2}$ 保护下, 将 $N, N$-二甲基甲酰胺(DMF)加入到 三颈瓶中, 然后用注射器加入 $\mathrm{POCl}_{3}$; 室温摚拌 $1 \mathrm{~h}$ 后加 入 I3T, 然后在 $75^{\circ} \mathrm{C}$ 下加热反应 $8 \mathrm{~h}$. 反应结束后, 加入 水充分水解, 然后用二氯甲烷萃取. 萃取液经水洗和无 水 $\mathrm{Na}_{2} \mathrm{SO}_{4}$ 干燥后, 减压蒸馏除去二氯甲烷得到粗产物. 用二氯甲烷作洗脱液, 粗产物经硅胶柱分离提纯得到淡 黄色晶体, 产率为 $70 \%$.

\section{4 电化学聚合}

单体 I3T 的电化学聚合是在 $0.1 \mathrm{~mol} \cdot \mathrm{L}^{-1} \mathrm{TBAP}$ 的乙 腈与乙醇混合溶液 $(V: V=9: 1)$ 中进行的; 单体 OHC-I3T-CHO 的电化学聚合是在 $0.1 \mathrm{~mol} \cdot \mathrm{L}^{-1} \mathrm{TBAP}$ 的 乙腈溶液中进行的. ITO 玻璃为工作电极、铂丝电极为 对电极、 $\mathrm{Ag} / \mathrm{Ag}^{+}\left(0.01 \mathrm{~mol} \cdot \mathrm{L}^{-1}\right)$ 为参比电极, 在 $-2.5 \sim$ $+2.5 \mathrm{~V}$ (vs. $\mathrm{Ag} / \mathrm{Ag}^{+}$) 的电位区间内, 以 $50 \mathrm{mV} / \mathrm{s}$ 的速率 进行扫描, 使 I3T 和 OHC-I3T-CHO 单体从支持电解质 中聚合到 ITO 玻璃上.

\section{2 结果和讨论}

\section{1 低聚噻吩衍生物单体的结构表征}

I3T: UV-vis $\left(\mathrm{CH}_{2} \mathrm{Cl}_{2}\right) \lambda_{\text {max }}: 244 \mathrm{~nm} ;{ }^{1} \mathrm{H}$ NMR (DMSO- $\left.d_{6}, 400 \mathrm{MHz}\right) \delta: 7.75$ (s, 2H, ArH), 7.53 (d, $J=3.9$ 
$\mathrm{Hz}, 2 \mathrm{H}, \mathrm{ArH}), 7.06$ (t, $J=4.1 \mathrm{~Hz}, 2 \mathrm{H}, \mathrm{ArH}), 6.99$ (d, $J=$ $3.9 \mathrm{~Hz}, 2 \mathrm{H}, \mathrm{ArH}$ ); ${ }^{13} \mathrm{C}$ NMR (DMSO- $\left.d_{6}, 400 \mathrm{MHz}\right) \delta$ : (DMSO- $\left.d_{6}, 400 \mathrm{MHz}\right), 137.51,134.49,128.17,127.55$, 127.15, 126.55; IR (KBr) v: $3096\left(v_{\mathrm{C}-\mathrm{H}}\right), 3067\left(v_{\mathrm{C}-\mathrm{H}}\right), 1663$ $\left(v_{\mathrm{C}=\mathrm{C}}\right) \mathrm{cm}^{-1}$; MS (APCI) $\mathrm{m} / \mathrm{z}: 249\left(\mathrm{M}^{+}\right)$.

OHC-I3T-CHO: UV-vis $\left(\mathrm{CH}_{2} \mathrm{Cl}_{2}\right) \lambda_{\max }: 312 \mathrm{~nm} ;{ }^{1} \mathrm{H}$ NMR $\left(\mathrm{CDCl}_{3}, 400 \mathrm{MHz}\right) \delta$ : 9.98 (s, 2H, CHO), 7.66 (d, $J=4.0 \mathrm{~Hz}, 2 \mathrm{H}, \mathrm{ArH}), 7.58$ (s, 2H, ArH), 7.01 (d, $J=4.0$ $\mathrm{Hz}, 2 \mathrm{H}, \mathrm{ArH}) ;{ }^{13} \mathrm{C}$ NMR $\left(\mathrm{CDCl}_{3}, 400 \mathrm{MHz}\right) \delta: 184.66$, 145.86, 143.52, 138.59, 132.66, 129.27, 129.21; IR (KBr) $v: 3063\left(v_{\mathrm{C}-\mathrm{H}}\right), 1723 \quad\left(v_{\mathrm{C}=\mathrm{O}}\right), 1656\left(v_{\mathrm{C}=\mathrm{C}}\right) \mathrm{cm}^{-1} ; \mathrm{MS}$ (APCI) $m / z: 305\left(\mathrm{M}^{+}\right)$.

\section{$2.2 \mathrm{OHC}-\mathrm{I} 3 \mathrm{~T}-\mathrm{CHO}$ 单体的单晶结构}

将纯净的 OHC-I3T-CHO 溶于乙腈中, 于室温下静 置, 缓慢挥发得到长方形单晶 (如图 1a 所示). 在室温下 取培养的单晶置于 $\mathrm{D} / \mathrm{max}$-IIIA 单晶衍射仪上, 用 $M o-K \alpha$ 辐射 $X$ 射线进行扫描. 单晶结构由 Shelxtl-97 进 行解析, 并进行全矩阵最小二乘法修正, 所有非氢原子 使用 SADABS 程序全部进行吸收校正. 图 $1 \mathrm{~b}$ 为 OHC-I3T-CHO 单体的晶体结构. 表 1 为 OHC-I3T-CHO 单体的单晶衍射数据. 结果表明, OHC-I3T-CHO 单体属 于正交晶系, $P b c a$ 空间群, 晶胞参数 $a=1.3589$ (3) $\mathrm{nm}, b$ $=1.3669$ (3) nm, $c=1.4468$ (4) nm, $V=2.6894$ (9) $\mathrm{nm}^{3}, Z$ $=8, D_{\mathrm{c}}=1.505 \mathrm{Mg} / \mathrm{m}^{3}, \mu=0.544 \mathrm{~mm}^{-1}, F(000)=1248$.

表 1 OHC-I3T-CHO 单体的单晶衍射数据

Table 1 Summary of structural information for OHC-I3T-CHO monomer

\begin{tabular}{ll}
\hline \multicolumn{1}{c}{ Parameter } & \multicolumn{1}{c}{ Data } \\
\hline Empirical formula & $\mathrm{C}_{14} \mathrm{H}_{8} \mathrm{O}_{2} \mathrm{~S}_{3}$ \\
Formula weight & 304.38 \\
Temperature/K & $293(2)$ \\
Wavelength $/ \AA$ & 0.71073 \\
$a / \mathrm{nm}$ & $1.3589(3)$ \\
$b / \mathrm{nm}$ & $1.3669(3)$ \\
$c / \mathrm{nm}$ & $1.4468(4)$ \\
$\alpha /\left(^{\circ}\right)$ & 90.00 \\
$\beta /\left(^{\circ}\right)$ & 90.00 \\
$\gamma /\left(^{\circ}\right)$ & 90.00 \\
Volume/nm & \\
$Z$ & $2.6874(9)$ \\
$F(000)$ & 8 \\
Calculated density $/\left({\left.\mathrm{Mg} \cdot \mathrm{m}^{-3}\right)}^{\text {Absorption coefficient } / \mathrm{mm}^{-1}}\right.$ & 1248 \\
\hline
\end{tabular}

\section{3 低聚噻吩衍生物单体的电化学聚合}

由于单一噻吩的氧化电位比较高, 如果用单一噻吩 作为单体进行电化学聚合制备聚噻吩薄膜, 可能会由于 施加的电压过高而出现过氧化现象, 导致所得薄膜的氧
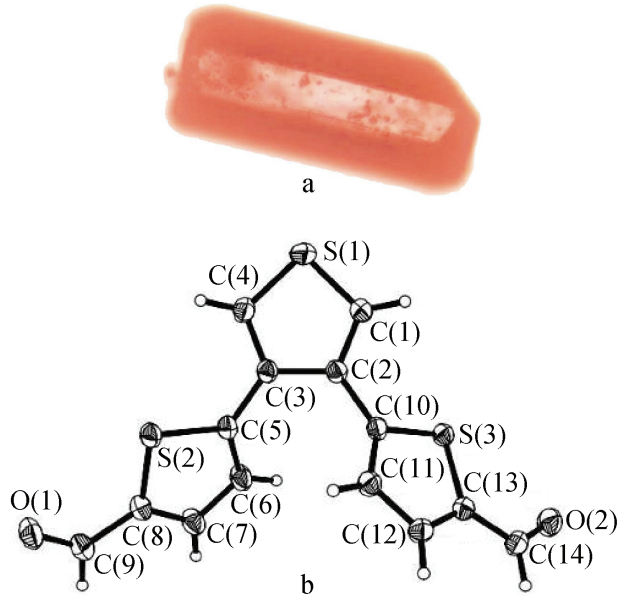

图 1 (a) OHC-I3T-CHO 单体的单晶偏光显微镜照片和(b) OHC-I3T-CHO 单体的晶体结构

Figure 1 (a) The monocrystal photomicrograph of OHC-I3T$\mathrm{CHO}$ monomer and (b) the crystal structure of OHC-I3T-CHO monomer

化还原性能下降 ${ }^{[7]}$, 而用 I3T 和 OHC-I3T-CHO 作为单体 进行电化学聚合制备聚噻吩衍生物薄膜可以克服这个 问题. 分别在 $0.1 \mathrm{~mol} \cdot \mathrm{L}^{-1} \mathrm{TBAP}$ 的乙腈与乙醇混合溶液 $(V: V=9: 1)$ 和 $0.1 \mathrm{~mol} \cdot \mathrm{L}^{-1} \mathrm{TBAP}$ 的乙腈溶液中, 单体 I3T $\left(0.1 \mathrm{~mol} \cdot \mathrm{L}^{-1}\right)$ 和 $\mathrm{OHC}-13 \mathrm{~T}-\mathrm{CHO}\left(0.05 \mathrm{~mol} \cdot \mathrm{L}^{-1}\right)$ 可在较 低的电压作用下发生电化学聚合, 得到稳定的聚噻吩衍 生物薄膜. 图 2 为单体 I3T 和 OHC-I3T-CHO 电化学聚 合的循环伏安曲线. 从图 2 可以看出, 在重复扫描的过 程中, 随着扫描次数的增加, 电流逐渐增加. 这个结果 说明, 单体 I3T 和 OHC-I3T-CHO 在 ITO 玻璃上发生了 聚合, 形成了聚(I3T)和聚(OHC-I3T-CHO) 薄膜.

\section{4 聚噻吩衍生物薄膜的电致变色性能}

\subsection{1 聚(I3T) 薄膜的电致变色性能}

将覆盖有聚(I3T)薄膜的 ITO 玻璃浸入到 $0.1 \mathrm{~mol} \cdot \mathrm{L}^{-1}$ TBAP 的乙腈与乙醇混合溶液 $(V: V=9: 1)$ 中, 以 ITO 玻璃和铂线作为电极, 考察聚(I3T)薄膜的电致变色性 能. 图 3a 为聚(I3T)薄膜在外加电压作用下的颜色变化 照片, 当在 ITO 电极和铂线电极之间施加 $+2.3 \mathrm{~V}$ 的电 压时, 聚(I3T)薄膜的颜色由淡黄色变为淡蓝色; 当外加 电压为 $-2.3 \mathrm{~V}$ 时, 聚(I3T)薄膜的颜色能发生可逆的变 化, 由淡蓝色变回淡黄色.

\subsection{2 聚(OHC-I3T-CHO) 薄膜的电致变色性能}

将覆盖有聚(OHC-I3T-CHO)薄膜的 ITO 玻璃浸入 到 $0.1 \mathrm{~mol} \cdot \mathrm{L}^{-1} \mathrm{TBAP}$ 的乙腈溶液中, 以 ITO 玻璃和铂线 作为电极, 考察聚 $(\mathrm{OHC}-\mathrm{I} 3 \mathrm{~T}-\mathrm{CHO})$ 薄膜的电致变色性 能. 图 $3 \mathrm{~b}$ 为聚 $(\mathrm{OHC}-\mathrm{I} 3 \mathrm{~T}-\mathrm{CHO})$ 薄膜在外加电压作用下 的颜色变化照片. 当在 ITO 电极和铂线电极之间施加 + $1.5 \mathrm{~V}$ 的电压时, 聚( $\mathrm{OHC}-\mathrm{I} 3 \mathrm{~T}-\mathrm{CHO})$ 薄膜的颜色由红 

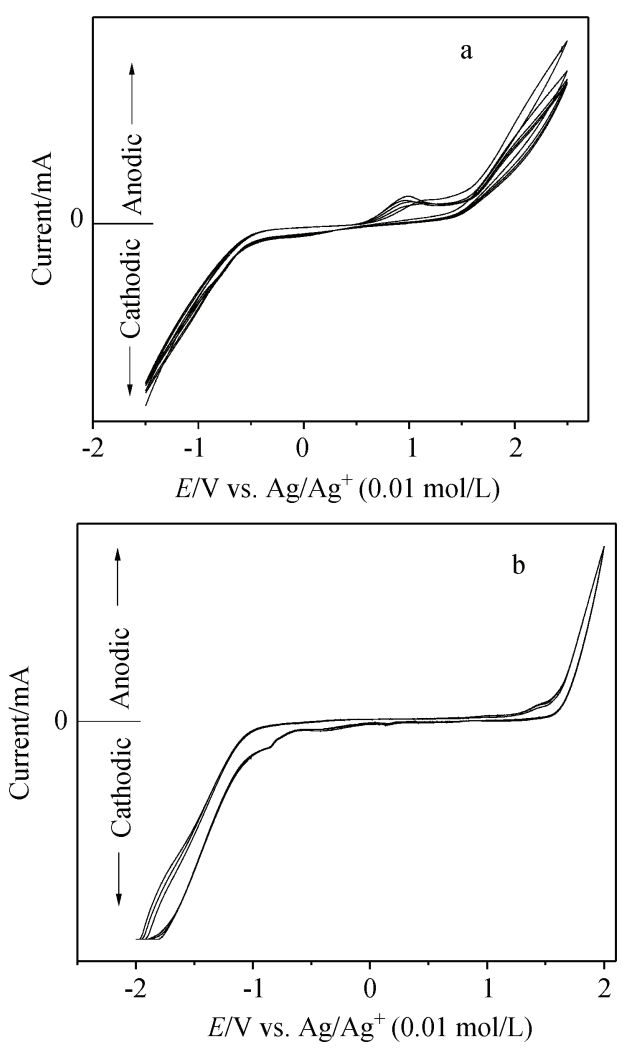

图 2 单体 I3T (a) 和 OHC-I3T-CHO (b)的电化学聚合循环伏安 曲线

Figure 2 Electropolymerization of I3T (a) and OHC-I3T-CHO (b) monomers on ITO electrode

色变为墨绿色; 当外加电压为 $-1.5 \mathrm{~V}$ 时, 聚 (OHC-I3T-CHO) 薄膜的颜色能发生可逆的变化, 由墨绿 色变回红色.

\section{3 结论}

合成了两种低聚噻吩衍生物 I3T 和 OHC-I3T-CHO. 以 I3T 和 OHC-I3T-CHO 作为单体, 通过电化学聚合制 备了相应的聚噻吩衍生物. 研究了聚噻吩衍生物薄膜的

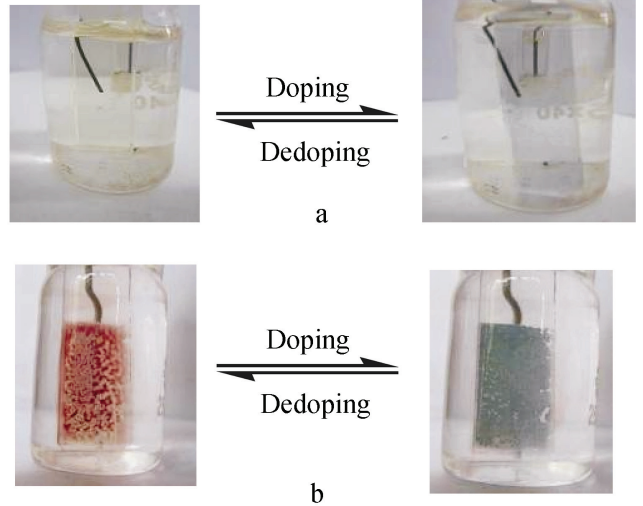

图 3 聚噻吩衍生物薄膜的电致变色照片

Figure 3 Photographs of the electrochromism of polythiophene derivative films

a: poly(I3T) film; b: poly(OHC-I3T-CHO) film

电致变色性能, 研究发现, 聚噻吩衍生物薄膜均具有好 的电致变色性能, 在外加电压作用下能发生稳定的可逆 颜色变化. 今后的工作是优化电致变色器件, 进一步了 解和掌握聚(I3T)和聚( $\mathrm{OHC}-\mathrm{I} 3 \mathrm{~T}-\mathrm{CHO})$ 薄膜的电致变色 机理, 为研制和开发新型有机电致变色器件奠定坚实的 基础.

\section{References}

1 Akoudad, S.; Roncali, J. Synth. Met. 1999, 101, 149.

2 Li, X. H.; Li, Y. F. Synth. Met. 2003, 135, 215.

3 Alkan, S.; Cutler, C. A.; Reynolds, J. R. Adv. Funct. Mater. 2003, 13, 331.

4 Pang, Y. H.; Li, X. Y.; Jin, H. T. Electrochim. Acta 2007, 52,6172 .

5 Kim, B. G.; Kim, J. H.; Kim, E. Y. Macromolecules 2011, 44, 8791 .

6 La, M.; Liu, M. M.; Liu, P.; Deng, W. J.; Tong, Z. Chin. J. Chem. 2008, 26, 1523.

7 Alkan, S.; Charlotte, A. C. Adv. Funct. Mater. 2003, 13, 331.

(A1109093 Cheng, B.) 